\title{
socs7, a target gene of microRNA-145, regulates interferon- $\beta$ induction through STAT3 nuclear translocation in bladder cancer cells
}

\author{
S Noguchi ${ }^{\star, 1}$, N Yamada ${ }^{1,2}$, M Kumazaki $^{1,3}$, Y Yasui ${ }^{1,3}$, J Iwasaki ${ }^{1,3}$, S Naito ${ }^{4}$ and Y Akao ${ }^{1}$
}

We recently reported that microRNA (miR)-145 is downregulated and induces apoptosis in human bladder cancer cells. Also, it is suggested that the ectopic expression of miR-145 induces apoptosis with the induction of TRAIL expression in several cancer cells. Here, we demonstrated a novel mechanism of apoptosis induction by miR-145 in bladder cancer cells. Exogenous miR-145 in T24 and NKB1 cells markedly increased the expression levels of interferon (IFN)- $\beta, 2{ }^{\prime}-5^{\prime}$-oligoadenylate synthetase 1 , which lies upstream of $2^{\prime}-5^{\prime}$ oligoadenylates/RNase $L$ system, and TRAIL, and induced apparent caspase-dependent apoptosis that was suppressed by cotreatment with a pan-caspase inhibitor; moreover, these expression levels were reduced by cotreatment with an miR-145 inhibitor. The apoptosis did not depend on Toll-like receptor 3 (TLR3) expression, because TLR3-silencing failed to inhibit IFN- $\beta$ induction by miR-145. Then, we focused on the suppressor of cytokine signaling 7 (socs 7 ), whose expression level was upregulated in bladder cancer cells compared with its level in normal human urothelial cells, as a putative target gene involved in IFN- $\beta$ induction by miR-145. Expectedly, exogenous miR-145 decreased the expression level of SOCS7, and socs7silencing enhanced IFN- $\beta$ induction by transfection with a TLR3 ligand, polyinosinic acid-polycytidylic acid (PIC). The results of a luciferase reporter assay revealed that miR-145 targeted socs7. In addition, socs7-silencing significantly decreased the level of p-Akt and suppressed the growth of T24 cells. Furthermore, exogenous miR-145 or socs7-silencing promoted nuclear translocation of STAT3. In conclusion, the machinery of IFN- $\beta$ induction through the regulation of SOCS7 by miR-145 was closely associated with the induction of apoptosis. Moreover, exogenous miR-145 promoted IFN- $\beta$ induction by targeting socs7, which resulted in the nuclear translocation of STAT3. Additionally, our data indicate that SOCS7 functioned as an oncogene, the finding that revealed a novel mechanism of carcinogenesis in bladder cancer cells.

Cell Death and Disease (2013) 4, e482; doi:10.1038/cddis.2013.11; published online 7 February 2013

Subject Category: Cancer

microRNA-145 (miR-145) has been frequently reported to be downregulated in various human cancers, including prostate cancer, ${ }^{1}$ bladder cancer, ${ }^{2}$ and colon cancer, ${ }^{3}$ as well as in B-cell malignancies. ${ }^{4}$ Moreover, we recently reported that miR-145 is downregulated in human bladder cancer cells and human melanoma cells. ${ }^{5-7}$ miR-145 functions as a tumor suppressor in various human cancers, and its target genes include $c$-myc, ${ }^{8}$ fascin $1,{ }^{9}$ yes, ${ }^{10}$ oct4, klf4$^{11}{ }^{11}$ and ilk. $^{7}$ Furthermore, Ostenfeld et al. ${ }^{12}$ reported that miR-145 induces caspase-dependent and -independent cell death in human urothelial cancer cells, and two other groups reported that the transfection of cells with miR-145 results in Tumor Necrosis Factor-related Apoptosis-Inducing Ligand (TRAIL)-induced apoptotic cell death. ${ }^{13,14}$ However, the detailed mechanism of the apoptosis induced by miR-145 remains unclear.
TRAIL is known to have a role in Mycobacterium bovis Bacillus Calmette-Guerin (BCG)-induced antitumor effects, in which, attenuated bacterium is generally used in the treatment of bladder cancer. ${ }^{15}$ The expression of TRAIL is induced by certain kinds of cytokines, for example, interferon (IFN)- $\beta .^{16}$ IFN- $\beta$ is secreted from cells in response to a viral infection or the introduction of double-stranded RNA (dsRNA), including siRNA. ${ }^{17,18}$ One of the most important pathways for IFN induction by dsRNA is the Toll-like receptor 3 (TLR3)/TLR adapter molecule 1 (TRIF) pathway. TLR3 recognizes dsRNA in the lumen of the endosome and activates the signaling cascade via TRIF. ${ }^{19}$ Viral infection or the introduction of dsRNA also induces activation of the $2^{\prime}-5^{\prime}$ oligoadenylates $(2-5 A) / R N a s e ~ L$ system, which is triggered by $2^{\prime}-5^{\prime}$-oligoadenylate synthetase 1 (OAS1). ${ }^{20}$ OAS1 is also recognized

\footnotetext{
${ }^{1}$ United Graduate School of Drug Discovery and Medical Information Sciences, Gifu University, 1-1 Yanagido, Gifu, Japan; ${ }^{2}$ United Graduate School of Veterinary Sciences, Gifu University, 1-1 Yanagido, Gifu, Japan; ${ }^{3}$ Graduate School of Engineering, Gifu University, 1-1 Yanagido, Gifu, Japan and ${ }^{4}$ Department of Urology, Graduate School of Medical Sciences, Kyushu University, 6-10-1 Hakozaki, Higashi-ku, Fukuoka, Japan

*Corresponding author: S Noguchi, United Graduate School of Drug Discovery and Medical Information Sciences, Gifu University, 1-1 Yanagido, Gifu 501-1193, Japan. Tel: + 81-58-230-7607; Fax: + 81-58-230-7604; E-mail: snoguchi@ gifu-u.ac.jp

Keywords: bladder cancer; interferon- $\beta$; microRNA-145; SOCS7; STAT3

Abbreviations: IFN, interferon; ilk, integrin-linked kinase; ISGs, IFN-stimulated genes; klf4, Krueppel-like factor 4; OAS1, 2'-5'-oligoadenylate synthetase 1; oct-4, octamer-binding transcription factor 4; PIC, polyinosinic acid-polycytidylic acid; RT-PCR, reverse transcription polymerase chain reaction; SAPK/JNK, activation of stress-activated protein kinase/jun amino-terminal kinase; siRNA, small interfering RNA; SOCS7, suppressor of cytokine signaling 7; STAT3, signal transducer and activator of transcription 3; TLR3, Toll-like receptor 3; TRAIL, tumor necrosis factor-related apoptosis-inducing ligand; TRIF, TLR adapter molecule 1; 2-52-5A, 2'-5' oligoadenylates

Received 05.12.12; revised 21.12.12; accepted 27.12.12; Edited by A Stephanou
} 
as one of the IFN-stimulated genes (ISGs), as is TRAIL. ${ }^{21}$ Binding of $2-5 A$ to RNase $L$ converts the enzyme from inactive monomers to active dimers. ${ }^{22}$ The sustained activation of RNase $L$ by $2-5 A$ leads to apoptosis through the activation of stress-activated protein kinase/jun amino-terminal kinase (SAPK/JNK) and mitochondrial pathways, ${ }^{20}$ thus limiting the spread of viral infections. ${ }^{23}$

On the other hand, the expression of the suppressor of cytokine signaling (SOCS) proteins is also induced by cytokine stimulation; ${ }^{24}$ and overexpression of SOCS proteins in various cell lines results in the inhibition of signaling by a wide range of cytokines. These findings indicate that SOCS proteins are also important for the regulation of IFN signaling pathways. Numerous studies have clarified the functions of SOCS1, SOCS2, and SOCS3 proteins. ${ }^{24}$ However, studies focused on the function of SOCS7 are limited. A recent study shows that SOCS7 attenuates nuclear translocation of STAT3 and STAT5. ${ }^{25}$ STAT3 is one of the important transcriptional factors that target genes for cytokines such as IFN- $\beta$ and interleukin $6 .^{26}$

This study demonstrates one of the mechanisms responsible for the induction of apoptosis in human bladder cancer cells transfected with miR-145. We also validated socs 7 as a possible target gene involved in apoptosis induced by exogenous miR-145. Furthermore, a novel function of SOCS7 was disclosed through the study of miR-145 and its target gene. We suggest that the downregulation of miR-145 contributes to carcinogenesis via one of its target genes, socs7, by regulating IFN- $\beta$-induced apoptosis, which is crucial for immune eradication of nonself.

\section{Results}

Transfection with miR-145 inhibited the growth of bladder cancer cells and resulted in caspase-dependent apoptotic cell death with the upregulation of IFN- $\beta$ and ISGs. As shown in Figure 1a, the introduction of miR-145 via transfection significantly inhibited the growth of all the bladder cancer cell lines tested, in which the expression level of miR-145 is known to be downregulated. ${ }^{7}$ The mRNA expression levels of IFN- $\beta$ and ISGs such as OAS1 and TRAIL were significantly upregulated by transfection of T24 and NKB1 cells with miR-145, not but in the transfected SNK57 cells (Figure 1b). We also examined the expression levels of IFN- $\alpha$ and IFN- $\gamma$ by real-time RT-PCR in T24 and NKB1 cells transfected with control miRNA or miR-145. However, the expression levels of these genes were under the detection limit (the Ct value >35; data not shown). Caspase-dependent apoptotic cell death, which was indicated by the appearance of the cleaved form of PARP-1 and the active form of caspase-8, was observed by the ectopic miR-145 expression in T24 and NKB1 cells, but not in SNK57 cells (Figure 1c). Consistently, the TLR3 expression level was increased by miR-145 transfection of T24 cells and NKB1 cells. Furthermore, the level of p-SAPK/JNK, which acts downstream of $2-5 A / R N a s e ~ L$ system, was markedly increased in T24 cells, not but in NKB1 and SNK57 ones. However, in SNK57 cells, which showed no evidence of apoptosis, the TLR3 expression level was the same for control miRNA- and miR-145-transfected cells (Figure 1c). These findings indicate that the induction of IFN- $\beta$ expression
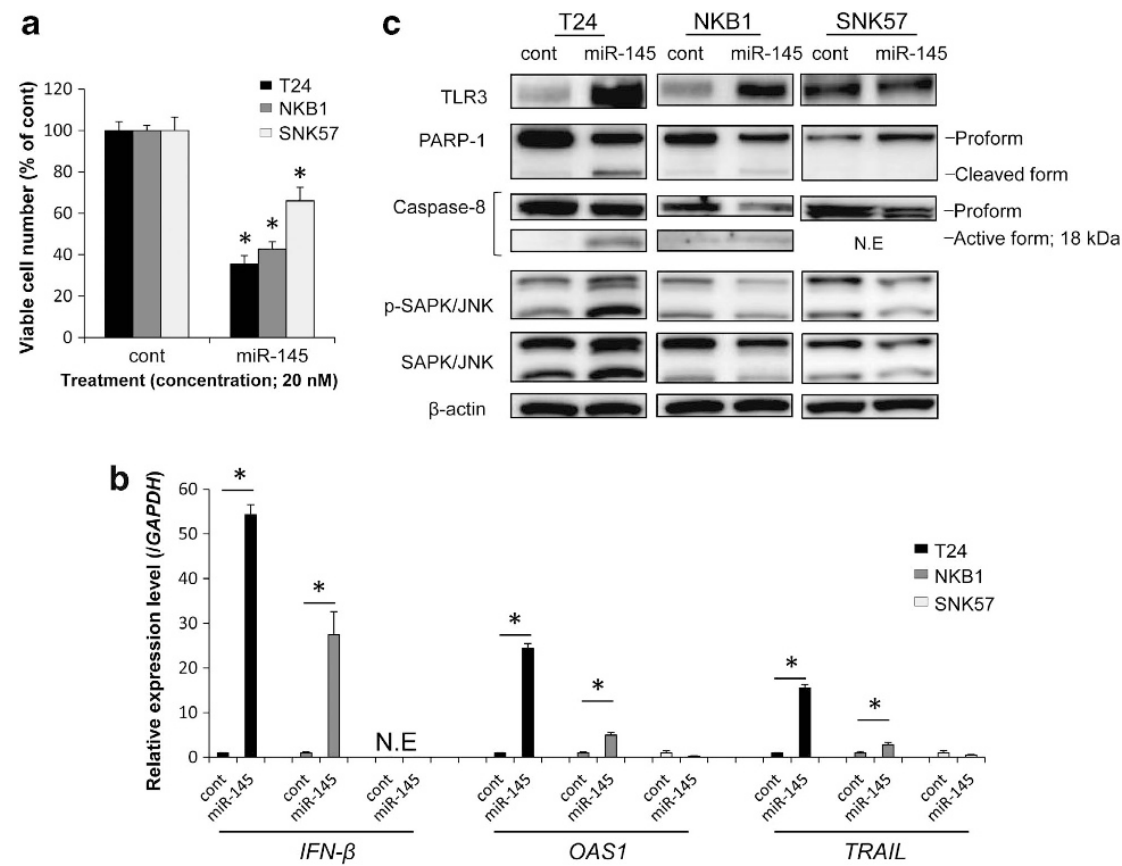

Figure 1 (a) Growth suppression of bladder cancer cells by transfection with miR-145. The viable cell number in the control is indicated as $100 \%$. ${ }^{*} P<0.01$. A $P$-value was determined for the difference between the cells transfected with control miRNA and those transfected with miR-145. (b) Expression levels of IFN- $\beta$ and ISGs by real-time PCR in bladder cancer cells transfected with miR-145. The expression level in the control is indicated as 1.0. N.E indicates 'not expressed'. * $P<0.01$. A $P$-value was determined for the difference between the cells transfected with control miRNA and those transfected with miR-145. (c) Protein expression levels in bladder cancer cells by western blotting analysis. Viable cell count and extraction of total protein and total RNA were performed at $72 \mathrm{~h}$ (T24) or $96 \mathrm{~h}$ (NKB1 and SNK57) after the transfection with control miRNA or miR-145 at a concentration of $20 \mathrm{nM}$. Data are expressed as the mean + S.D. $(n=3)$ 
by exogenous miR-145 contributed to the induction of apoptosis. In subsequent experiments, we mainly focused on T24 cells, because the most obvious caspase-dependent apoptosis triggered by exogenous miR-145 was observed in them (Figure 1c).

TLR3 silencing resulted in no suppression of IFN- $\beta$ induction by exogenous miR-145, and PIC transfectioninduced levels of IFN- $\beta$ and ISGs were greater than those by miR-145 transfection in T24 cells. Initially, we hypothesized that TLR3 contributed to IFN- $\beta$ induction by miR-145. Therefore, to test the validity of this hypothesis we performed TLR3 silencing in T24 cells by using siRNA. However, the expression of IFN- $\beta$ induced by miR-145 was not significantly suppressed (Figure 2a). Furthermore, the level of OAS1 expression and that of PARP-1 cleavage were almost unchanged, in spite of the marked reduction in TLR3 expression (Figures $2 a$ and $b$ ).

To compare the degree of IFN- $\beta$ induction by PIC or miR145 transfection, we also examined the expression levels of IFN- $\beta, O A S 1$, and TRAIL, and caspase-dependent apoptosis in T24 cells transfected with PIC at a concentration of $20 \mathrm{nM}$, which was the same dose used for the miR-145 transfection. As shown in Figure 2c, transfection of the cells with PIC markedly upregulated the expression levels of IFN- $\beta$, OAS1, and TRAIL. Those effects by PIC transfection were much greater than those achieved with exogenous miR-145 (cf., Figures $1 b$ and 2c). Moreover, cleaved PARP-1 and activated caspase-8 were also detected (Figure 2d). Additionally, the expression level of TLR3 and the level of $p$-SAPK/JNK were increased.

SOCS7 was upregulated, and miR-145 targeted socs7 in bladder cancer cells. We focused on socs7 as a possible target gene associated with IFN- $\beta$ induction by miR-145, because IFN- $\beta$ expression is reportedly modulated by the SOCS proteins. ${ }^{24}$ The SOCS7 expression level in T24 and NKB1 cells was upregulated compared with that in HUC (Figure 3a), and the ectopic expression of miR-145-suppressed SOCS7 protein expression (Figure 3b). On the other hand, the expression level of socs7 mRNA in the T24 cells was increased by transfection with miR-145 (Figure 3c). Furthermore,the luciferase activity of T24 cells cotransfected with miR-145 and pMIR-socs7 wild was significantly inhibited, compared with that of the cells cotransfected with control siRNA and the sensor vector (Figure 3d). Mutations of the socs73'-UTR-binding site significantly reduced the ability of miR-145 to regulate luciferase expression compared with that for pMIR-socs 7 wild (Figure $3 d$ ). These results demonstrate that miR-145 directly targeted socs7 in bladder cancer T24 cells.
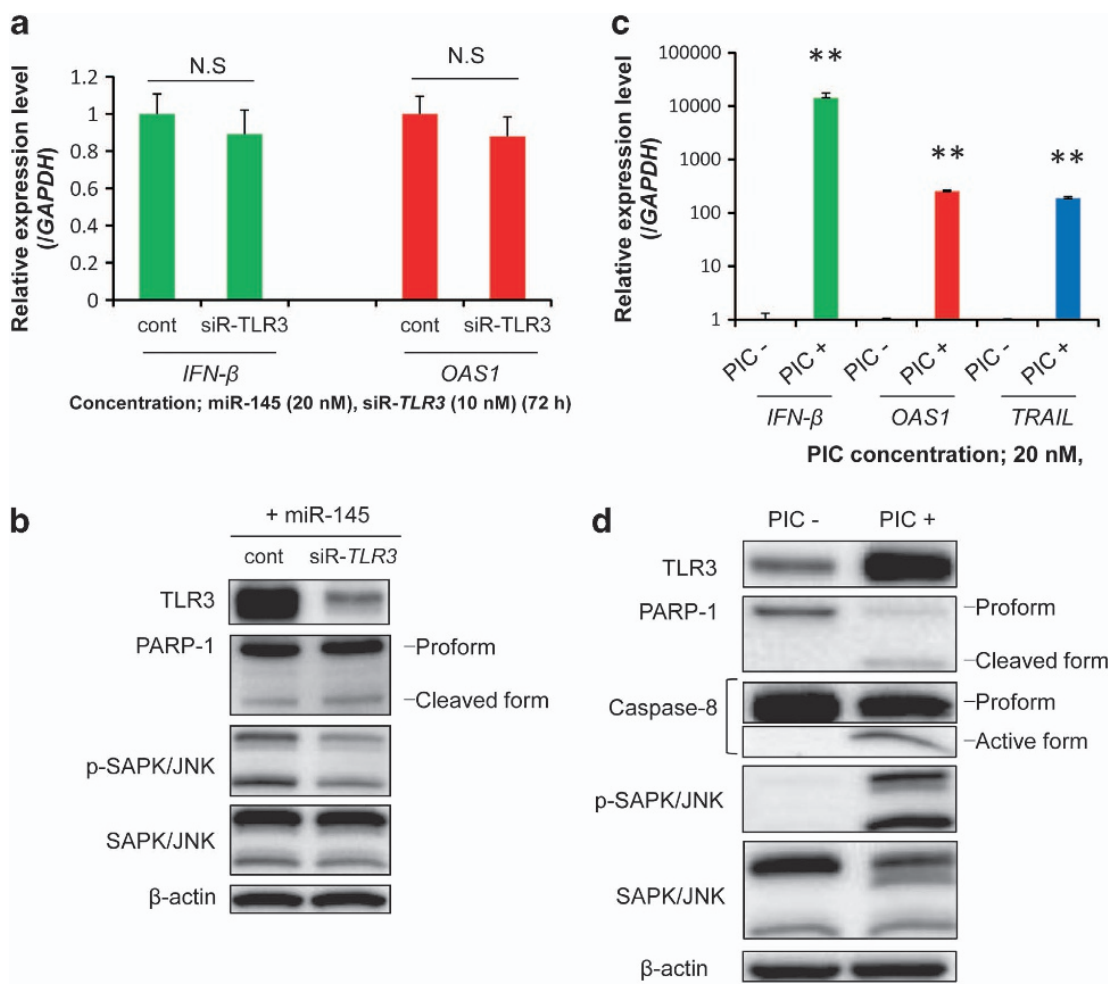

Figure 2 (a) Expression levels of IFN- $\beta$ and OAS1 in transfected T24 cells. The cells were cotransfected with miR-145 and control siRNA or siR-TLR3. The expression level in the control is indicated as 1.0. N.S indicates 'not significant'. ${ }^{*} P<0.05$. A $p$-value was determined for the difference between the cells cotransfected with control siRNA and miR-145 and those cotransfected with siR-TLR3 and miR-145. (b) Protein expression levels in transfected T24 cells, as determined by western blotting. (c) Expression levels of IFN- $\beta$ and ISGs in T24 cells, as determined by real-time PCR. The expression level in the control is indicated as 1.0. ${ }^{* *} P<0.01$. A $P$-value was determined for the difference between the cells transfected with transfection reagent alone and those transfected with PIC. (d) Protein expression levels in T24 cells, as analyzed by western blotting. Total protein and total RNA were extracted at $72 \mathrm{~h}$ after the cotransfection with miR-145 (20 nM) and control siRNA or siR-TLR3 (10 nM) or $24 \mathrm{~h}$ after the transfection with PIC at a dose of $20 \mathrm{nM}$. Data are expressed as the mean + S.D. $(n=3)$ 


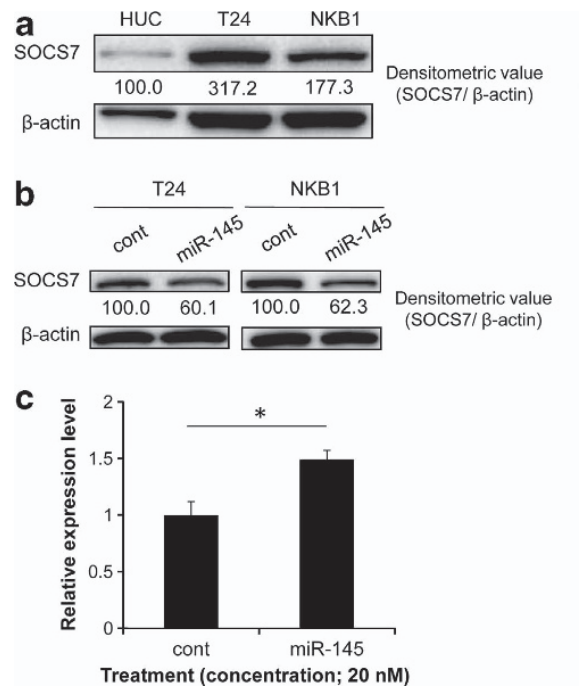

d
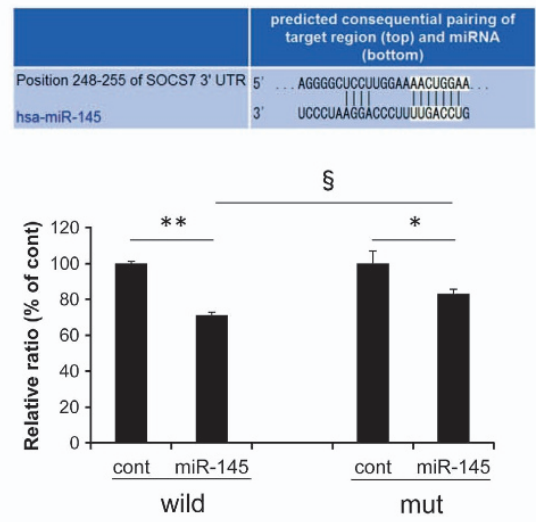

Figure 3 (a) SOCS7 protein expression in HUC and bladder cancer cells. (b) SOCS7 protein expression level after transfection with miR-145. (c) socs7 mRNA expression level after transfection of T24 cells with miR-145. The control expression level is indicated as 1.0. ${ }^{*} P<0.05$. A $P$-value was determined for the difference between the control miRNA- and miR-145-transfected cells. (d) The complementary regions of the $3^{\prime}$-UTR of human socs $7 \mathrm{mRNA}$ and mature miR-145 (upper panel) and luciferase activities after cotransfection with control miRNA or miR-145 and each of the sensor vectors having the $3^{\prime}$-UTR of socs7 (lower graph). ${ }^{*} P<0.05$, ${ }^{* *}$ and ${ }^{\S} P<0.01$. A $P$-value was determined for the difference in luciferase activity between the cells transfected with control miRNA and those transfected with miR-145 $\left({ }^{*}\right.$ and $\left.{ }^{* *}\right)$ or between the cells co-transfected with miR-145 and pMIR-socs 7 wild or pMIR-socs $7 \mathrm{mt}^{(\$)}$. The luciferase activity in the control is indicated as $100 \%$. Total protein and total RNA were extracted at $72 \mathrm{~h}$ (T24) or $96 \mathrm{~h}$ (NKB1) after the transfection with control miRNA or miR-145 at a concentration of $20 \mathrm{nM}$. Data are expressed as the mean + S.D. $(n=3)$

Cotreatment with miR-145 and miR-145 inhibitor or caspase inhibitor obviously inhibited the expression of IFN- $\beta$ and ISGs and/or lessened apoptosis. To show that the induction of IFN- $\beta$ and apoptosis by miR-145 was specific, we examined whether such induction would be inhibited by the cotreatment with an miR-145 inhibitor. As shown in Figure $4 a$, cotransfection with miR-145 and the miR-145 inhibitor attenuated the upregulation of IFN- $\beta$, OAS1, and TRAIL observed when miR-145 was used alone. Consistent with these findings, apoptosis and the cell growth-suppressing activities elicited by exogenous miR145 were also decreased by the added miR-145 inhibitor (Figures $4 b$ and $c$ ).

To ascertain that IFN- $\beta$ and TRAIL contribute to the apoptosis induced by miR-145, we also examined whether cotreatment with miR-145 and a caspase inhibitor, z-vad-fmk attenuates the apoptosis. Expectedly, z-vad-fmk partly canceled the growth suppression and suppressed apoptosis induced by exogenous miR-145 in T24 and NKB1 cells (Figures 4d and e).

IFN- $\beta$ upregulation and apoptosis by PIC were enhanced by socs 7 silencing. In a preliminary experiment, we determined the dose of PIC that would induce low-grade apoptosis in both T24 and NKB1 cells. As a result, the doses of $1 \mathrm{nM}$ (T24) and $3 \mathrm{pM}$ (NKB1) for PIC transfection were found to meet this requirement. Then, we examined the effects on the expression levels of IFN- $\beta, O A S 1$, and TRAIL in the cells when they were cotransfected with control siRNA or siR-socs7 and $1 \mathrm{nM}$ or $3 \mathrm{pM}$ of PIC. As a result, the expression level of IFN- $\beta$ in the cells cotransfected with siR-socs7 and PIC was significantly increased compared with that when control siRNA and PIC were used (Figure 5a).
The OAS1 expression level tended to increase and the $T R A I L$ expression level remained almost unchanged in T24 cells cotransfected with siR-socs7 and PIC compared with those in the case of cotransfection with control siRNA and PIC. On the other hand, cotransfection with siR-socs 7 and PIC significantly upregulated the expression levels of OAS1 and TRAIL in NKB1 cells (Figure 5a). Also, consistent with the upregulation of IFN- $\beta$, PARP-1 cleavage was enhanced by socs7-silencing in both T24- and NKB1 PIC-transfected cells (Figure $5 b$ ).

socs7-silencing inhibited the growth of bladder cancer cells by suppressing PI3K/Akt signaling. We also evaluated the effect of SOCS7 on the growth of bladder cancer cells by using siRNA for socs7. socs7-silencing significantly inhibited the growth of T24 cells and tended to suppress that of NKB1 cells (Figure 6a). The level of p-Akt was decreased in both T24 and NKB1 cells (Figure 6b). However, socs7silencing did not induce apoptosis or affect the levels of p-ERK1/2 and ERK1/2.

Exogenous miR-145 or socs7-silencing promoted the translocation of STAT3 into the nucleus. Next, by using the immunofluorescence method, we examined the intracellular localization of STAT3, which is one of the transcriptional factors for cytokines. As shown in Figure 7a, when T24 cells were transfected with control siRNA or cotransfected with control siRNA and PIC, the fluorescence was mainly distributed in the cytoplasm. On the other hand, in the cells transfected with miR-145 or cotransfected with siR-socs7 and PIC, the fluorescence was diffusely distributed in both the cytoplasm and nucleus. Especially, in the cells cotransfected with siR-socs7 and PIC, the fluorescence in the 

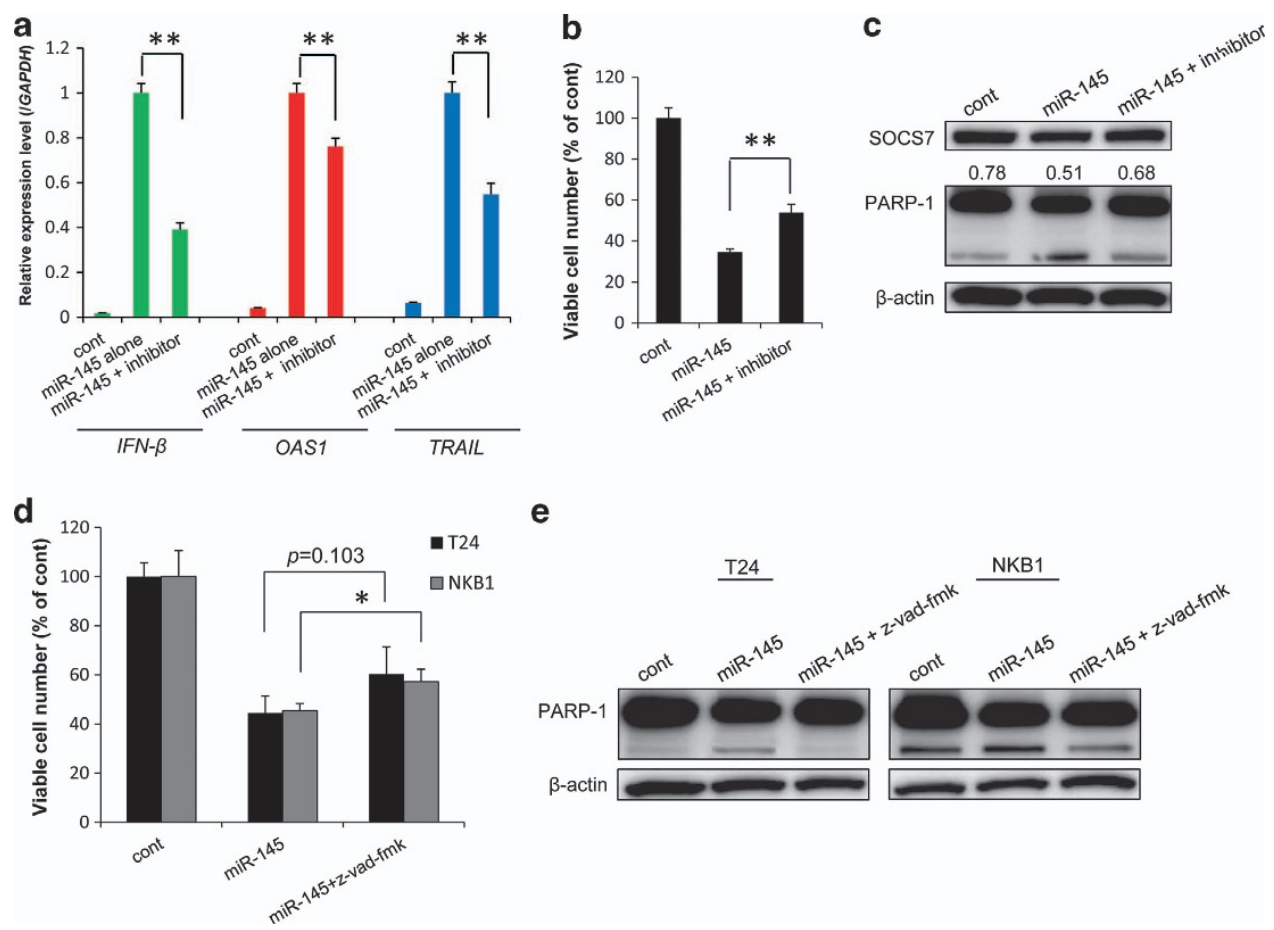

e

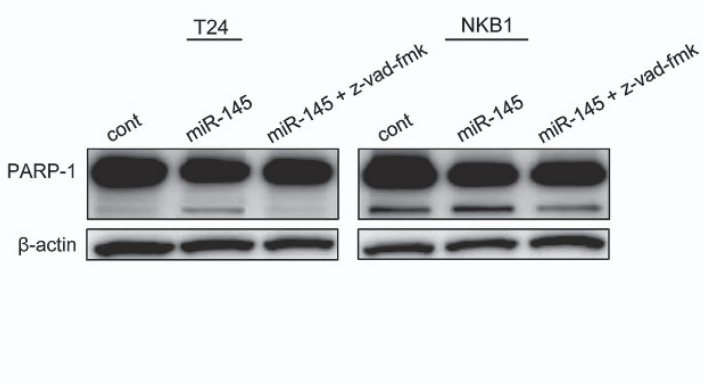

Figure 4 (a) Expression levels of IFN- $\beta$ and ISGs in T24 cells cotransfected with miR-145 and miR-145 inhibitor, as determined by real-time PCR. The expression level in the control is indicated as 1.0. ${ }^{*} P<0.01$. A $P$-value was determined for the difference between the cells transfected with miR-145 alone and those cotransfected with miR-145 and miR-145 inhibitor. (b) miR-145 inhibitor-mediated rescue of the growth of T24 cells transfected with miR-145. (c) Attenuation by miR-145 inhibitor of apoptosis in T24 cells transfected with miR-145. Densitometric value of SOCS7/ $\beta$-actin is expressed under the SOCS7 expression panel. (d) A pan-caspase inhibitor, $z$-vad-fmk partly canceled the growth suppression of T24 and NKB1 cells by exogenous miR-145. (e) Attenuation of apoptosis by z-vad-fmk in T24 and NKB1 cells transfected with miR-145. The viable cell number in the control is indicated as $100 \%$. ${ }^{*} P<0.05,{ }^{*} P<0.01$. A $P$-value was determined for the difference between the cells transfected with miR-145 alone and those cotransfected with miR-145 and miR-145 inhibitor or co-treatment with miR-145 and z-vad-fmk. Data are expressed as the mean + S.D. $(n=3)$. Extraction of total RNA and total protein and counting of the viable cells were performed at $72 \mathrm{~h}$ after the transfection with miR-145 (20 nM) and/or miR-145 inhibitor (40 nM)

nucleus was stronger than that in the cytoplasm. On the other hand, the transfection with siR-socs7 alone did not affect STAT3 localization (Supplementary Figure 1a). Such findings were also observed in the case of the NKB1 cells (Figure 7c).

We also examined the expression level of p-STAT3 by western blotting analysis. As a result, p-STAT3 level was markedly decreased in T24 cells transfected with miR-145 (Figure 7b). On the contrary, exogenous miR-145 slightly increased p-STAT3 level in NKB1 cells. On the other hand, socs7-silencing combined with PIC treatment increased p-STAT3 level in T24 and NKB1 cells (Figures 7b and d).

\section{Discussion}

In the current study, we successfully demonstrate a part of the mechanism of the induction of IFN- $\beta$ and apoptosis by miR-145 in bladder cancer cells. Type-1 IFNs are important therapeutic agents for the treatment of human bladder cancer, because IFN combined with BCG may salvage some bladder cancer patients, single-course BCG failures or late relapsers. ${ }^{27}$ To date, it has been indicated that the biochemical mechanism of apoptosis induction by miR-145 involves the induction of TRAIL expression. ${ }^{13}$ Data obtained in our present study suggest that IFN- $\beta$ induction, but not IFN- $\alpha$ and IFN- $\gamma$, subsequent activation of the $2-5 \mathrm{~A} / \mathrm{RNase} \mathrm{L}$ system, and upregulation of TRAIL have a central role in apoptotic cell death caused by exogenous miR-145. However, the $2-5 A / R N a s e ~ L$ system may not have contributed to apoptosis elicited by exogenous miR-145 in NKB1 cells, because no increase in activated SAPK/JNK was observed in these cells. Incidentally, upregulation of the above genes and caspasedependent apoptotic cell death by the ectopic expression of miR-145 was also observed in human melanoma A2058 cells (Supplementary Figures $2 \mathrm{a}$ and $\mathrm{b}$ ). We also attempted to examine the IFN- $\beta$ expression in the media by using ELISA kit and unfortunately could not detect the expression (data not shown). This finding indicates that IFN- $\beta$ was immediately faded from the media by autocrine and/or paracrine. Initially, we hypothesized that the induction of IFN- $\beta$ by exogenous miR-145 would depend on TLR3, because the expression level of TLR3 was markedly increased when T24 and NKB1 cells were transfected with miR-145, which cells showed an apparent caspase-dependent apoptosis. However, TLR3silencing clarified that TLR3 was not involved in IFN- $\beta$ induction by miR-145. The results of treatment with $20 \mathrm{nM}$ $\mathrm{PIC}$ also confirmed these findings, because the transfection with PIC showed a much greater degree of IFN- $\beta$ induction than the transfection with miR-145. Other IFN inducers, such as retinoic acid-inducible gene I (RIG-I) and melanoma differentiation-associated protein 5 (MDA5), can also recognize dsRNA. ${ }^{28}$ However, RIG-I recognizes longer dsRNAs, 

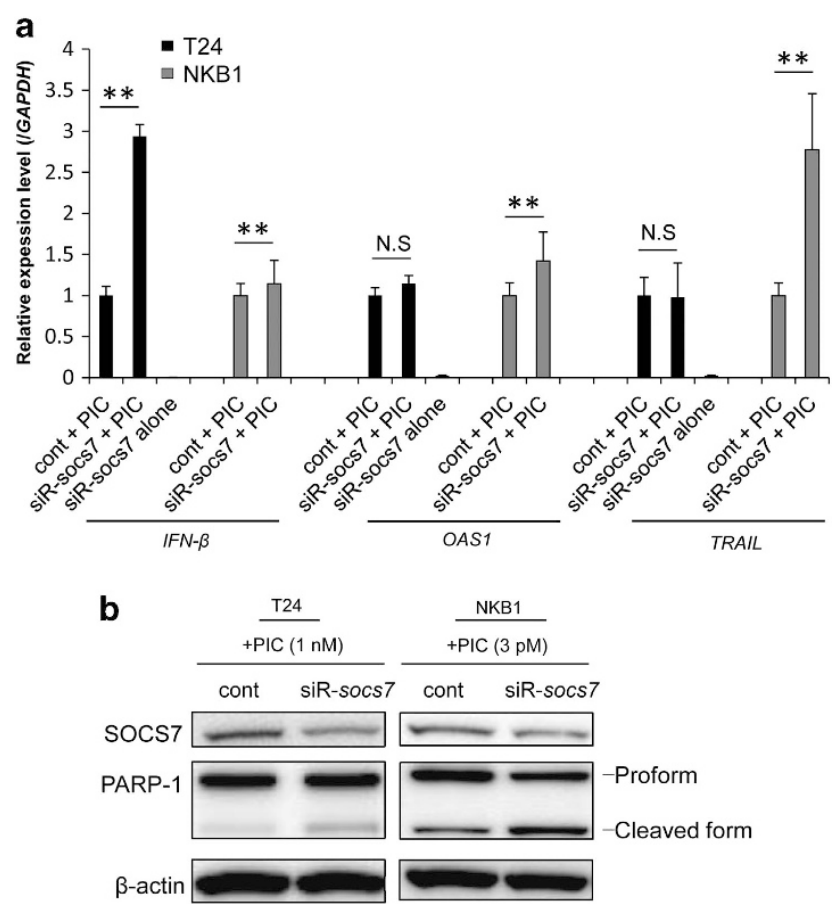

Figure 5 (a) Expression levels of IFN- $\beta$ and ISGs in T24 and NKB1 bladder cancer cells, as determined by real-time PCR. The expression level in the control is indicated as 1.0. N.S indicates 'not significant'. ${ }^{\star} P<0.05,{ }^{* \star} P<0.01$. A $P$-value was determined for the difference between the cells cotransfected with control siRNA and PIC and those cotransfected with siR-SOCS7 and PIC. (b) Western blot analysis of protein expression levels in the cells. The cells were transfected with PIC $(1 \mathrm{nM}, \mathrm{T} 24 ; 3 \mathrm{pM}, \mathrm{NKB} 1)$ at $48 \mathrm{~h}$ (T24) or $72 \mathrm{~h}$ (NKB1) after the transfection with control siRNA or siR-socs7 $(20 \mathrm{nM})$. Total RNA and total protein were extracted at $24 \mathrm{~h}$ after the transfection with PIC a

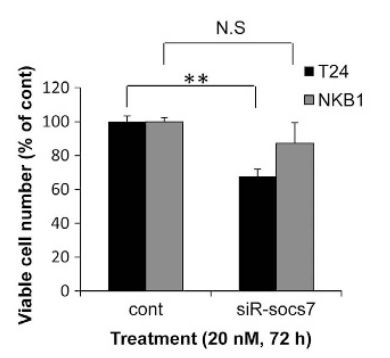

b

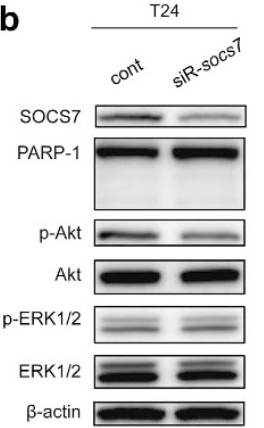

NKB1

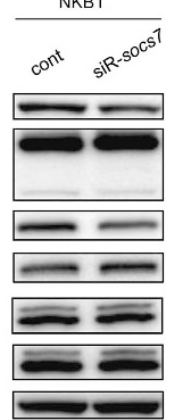

Figure 6 (a) Growth suppressing effect of socs7-silencing on T24 and NKB1 bladder cancer cells. (b) Protein expression levels in the cells, as determined by western blotting. Viable cell count and total protein extraction were performed at $72 \mathrm{~h}$ (T24) or $96 \mathrm{~h}$ (NKB1) after the transfection. The viable cell number in the control is indicated as $100 \%$. N.S indicates 'not significant'. ${ }^{*} P<0.01$. A $P$-value was determined for the difference between the cells transfected with control siRNA and those transfected with siR-socs7. Data are expressed as the mean + S.D. $(n=3)$

and MDA5 is associated with the TLR3 pathway. ${ }^{29,30}$ Accordingly, we consider that induction of IFN- $\beta$ and apoptosis by exogenous miR-145 was not mediated by RIGI and/or MDA5. Thus, we concluded that the induction of IFN- $\beta$ expression and apoptosis resulting from miR-145 transfection was not associated with some nonspecific effect of the introduced dsRNA.

We identified socs 7 as an miR-145 target gene involved in the IFN- $\beta$ signaling pathway in bladder cancer cells, based on the results of the luciferase reporter assay. socs7-silencing in PIC-transfected bladder cancer cells significantly enhanced the induction of IFN- $\beta$ expression and apoptosis. As the expression levels of OAS1 and TRAIL in T24 cells were not upregulated by cotreatment with PIC and siR-socs7, we consider that the IFN- $\beta$ expression level reached by PIC treatment alone had induced maximum expression levels of those genes. However, the treatment with siR-socs7 alone failed to induce IFN- $\beta$. These findings suggest that SOCS7 negatively regulated IFN- $\beta$ induction by exogenous miR-145, though SOCS7 was not directly associated with transcription of IFN- $\beta$. Actually, these findings also were observed in the case of human melanoma A2058 cells (Supplementary Figure 2c), in which miR-145 is downregulated. ${ }^{6}$ Importantly, the SOCS7 expression level was upregulated in T24 and NKB1 cells compared with that in HUC. Furthermore, socs7silencing inhibited the growth of bladder cancer cells by suppressing PI3K/Akt. These findings indicate that socs 7 acts as a possible oncogene by regulating the PI3K/Akt signaling pathway in bladder cancer cells. In a recent study, we showed that miR-145 regulates the PI3K/Akt signaling pathway in bladder cancer cells by targeting $i / k^{7}$ This study revealed that miR-145 also regulates this signaling pathway by targeting socs7. The function of SOCS7 has remained unclear compared with that of other SOCS proteins. However, our present study revealed that SOCS7 under stimulation of IFN- $\beta$ regulated STAT3 nuclear translocation. This finding supports the previous report by Martens et al. ${ }^{25}$ Commonly, STAT3 continuously shuttles between nuclear and cytoplasmic compartments, and nuclear translocation of STAT3 is independent of tyrosine phosphorylation, unlike other STATs. ${ }^{31}$ Importantly, both phosphorylated and unphosphorylated STAT3 upregulate IFN- $\beta$ expression. ${ }^{26}$ In our data, the level of p-STAT3 was not consistent by exogenous miR-145 in T24 and NKB1 cells, whereas socs7-silencing combined with PIC treatment consistently increased the p-STAT3 level in both cells. These findings indicate that nuclear translocation of STAT3 is independent of phosphorylation of STAT3, consistent with the previous study. ${ }^{31}$ To understand the comprehensive correlation with STAT3 and miR-145, the expression levels of other STATs such as STAT1 need to be examined, because p-STAT3 can heterodimerize with other phosphorylated STATs. Accordingly, we consider that miR-145 enhanced IFN- $\beta$ induction by inhibiting SOCS7, thus allowing the nuclear translocation of STAT3.

In conclusion, exogenous miR-145 induced IFN- $\beta$ expression, leading to the subsequent activation of $2-5 A / R N a s e ~ L$ system and upregulation of TRAIL and resulting in apoptotic cell death in human bladder cancer cells. We suggest that this mechanism has a central role in apoptosis induced by exogenous miR-145 and that this miRNA never has an offtarget effect. Additionally, our data show that miR-145 enhanced IFN- $\beta$ induction by itself through targeting socs7, which resulted in nuclear translocation of STAT3 and that SOCS7 contributed to the growth of bladder cancer cells via 
a $\underline{\mathrm{T} 24}$
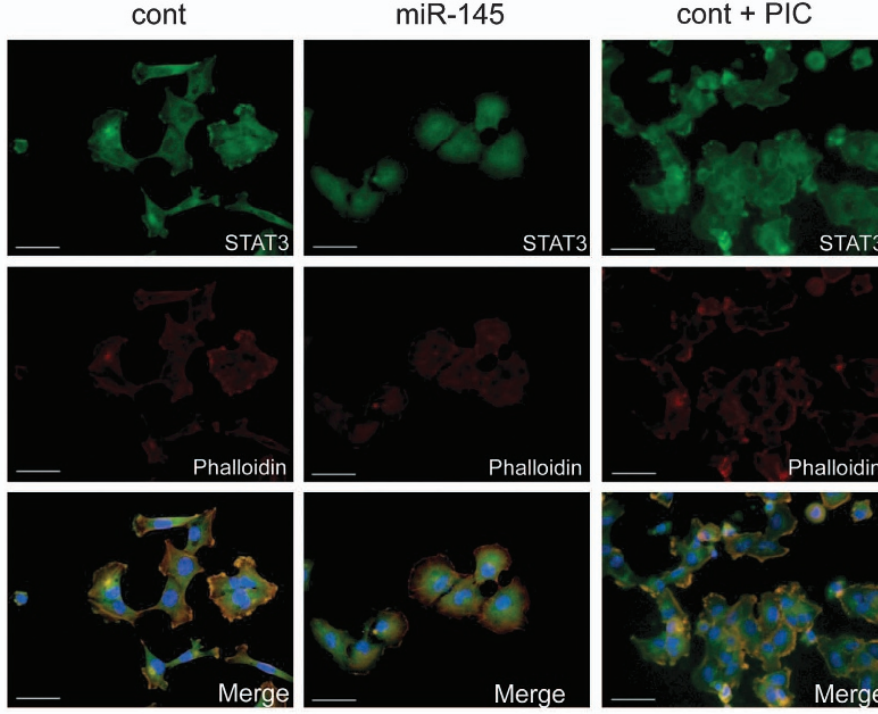

cont + PIC

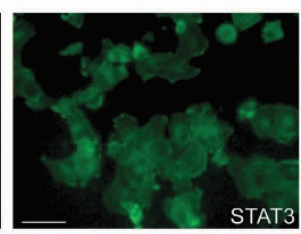

siR-socs7+ PIC
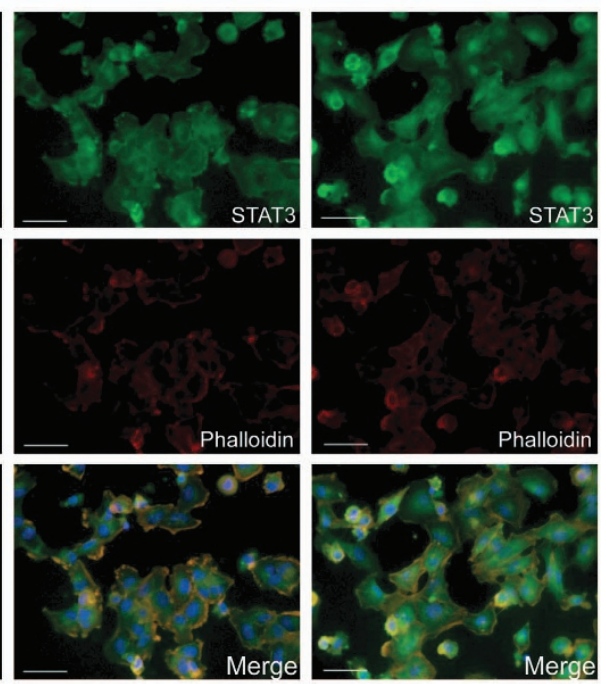

(miR-145 or siRNA; 20 nM, PIC; $1 \mathrm{nM}$ )

b
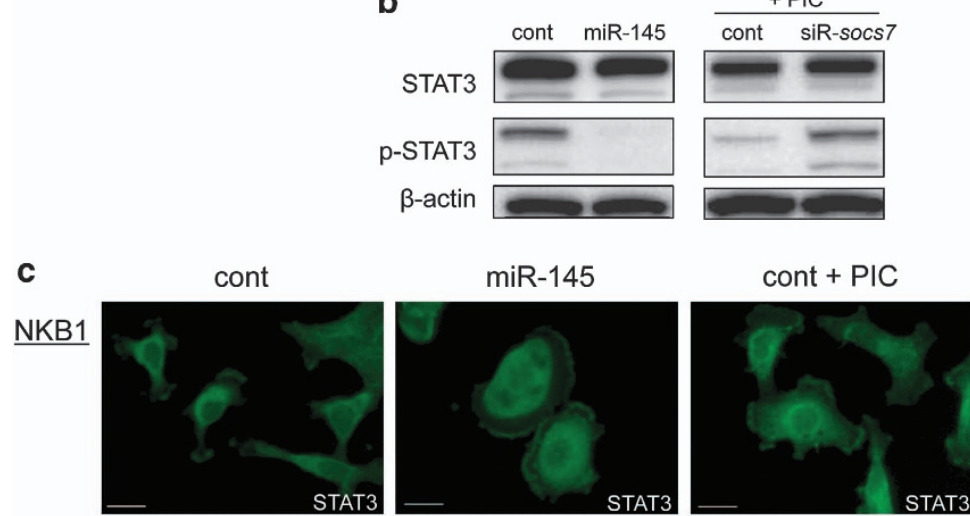

siR-socs7+ PIC
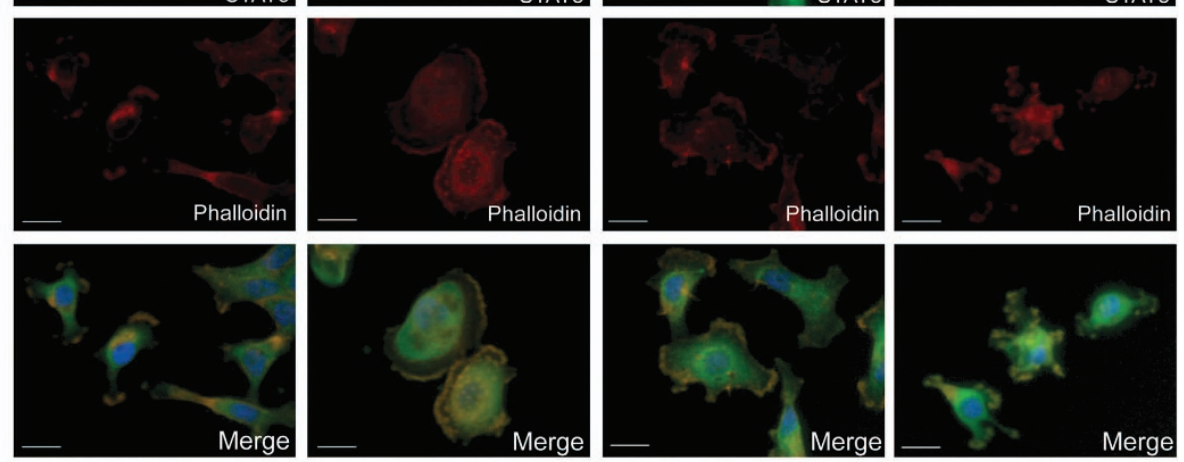

(miR-145 or siRNA; 20 nM, PIC; 3 pM)

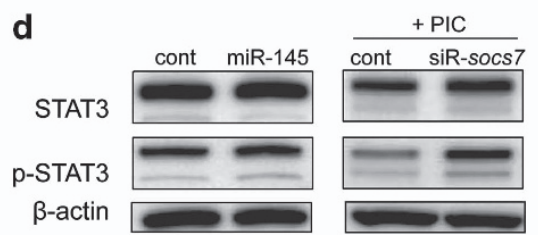

Figure 7 Intracellular distribution of STAT3 and the expression levels of STAT3 and p-STAT3 in T24 (a and $\mathbf{b})$ and NKB1 cells (c and d), respectively. Immunostaining by the immunofluorescence method was performed at $72 \mathrm{~h}$ after the transfection with control miRNA or miR-145. PIC was transfected for $24 \mathrm{~h}$ before the staining. Nuclei were counterstained in blue with Hoechst33342. Bar $=50 \mu \mathrm{m}$ (a) or $20 \mu \mathrm{m}$ (b) 


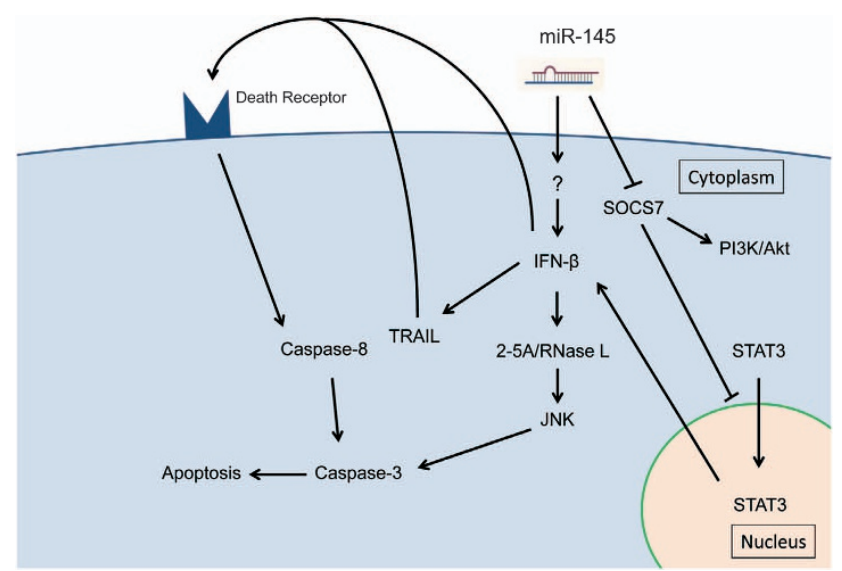

Figure 8 Flow diagram of the target genes of miR-145 and the related apoptosis inducing pathways

activation of $\mathrm{PI} 3 \mathrm{~K} / \mathrm{Akt}$ signaling pathway (Figure 8). This study successfully demonstrated a part of socs7 function, which had remained unclear until now, through analysis of miR-145 antioncogenic function. However, this study did not clear the initiator of IFN- $\beta$ by exogenous miR-145. Therefore, further studies need to be performed to define the comprehensive mechanism of IFN- $\beta$ induction by miR-145.

\section{Materials and Methods \\ Reagents. The mature type of miR-145 used for transfection in this study was purchased from Ambion (Foster City, CA, USA) and used at a concentration of $20 \mathrm{nM}$. miRIDIAN Hairpin Inhibitor, Human hsa-miR-145 (Thermo Scientific, Waltham, MA, USA) was used as an miR-145 inhibitor at a concentration of $40 \mathrm{nM}$. The siRNA for socs7 (siR-socs7; 5'-UGACAUUGCUGAAUCGGGACA CUGG-3') and that for TLR3 (siR-TLR3; 5'-AAUAAAUGGGACCACCAGG GUUUGC- $3^{\prime}$ ) were purchased from Invitrogen (Carlsbad, CA, USA). These siRNAs were used at a concentration of 10 or $20 \mathrm{nM}$. The nonspecific control siRNA was obtained from Thermo Scientific. Primers for TRAIL and socs7 were as follow: TRAlL-sense-91, 5'-GCTGACTTACAGCAGTCAGA-3', TRAIL-antisense-257, 5'-ATCTGCTTCAGCTCGTTGGT-3'; socs7-sense-1971, 5'-ACAGGAAGGTTGGG ATTCTC-3', and socs7-antisense-2330, 5'-CAGCACAGACTCTAACTCTG-3'. Primers for IFN- $\beta$ and OAS1 were obtained from Invivogen (San Diego, CA, USA). PIC, which is a TLR3 ligand, was purchased from Pharmacia Biotech (Piscataway, NJ, USA) and used to transfect cells for IFN- $\beta$ induction. A pan-caspase inhibitor, $z$-vad-fmk was purchased from Medical and Biological Laboratories (MBL;} Nagoya, Japan) and used at $50 \mu \mathrm{M}$.

Antibodies. Antibodies against phospho-ERK1/2 (p-ERK1/2), ERK1/2, phospho-Akt (Ser473; p-Akt), Akt, phospho-SAPK/JNK (Thr-183/Tyr-185; p-SAPK/JNK), SAPK/JNK, TLR3, STAT3, and phospho-STAT3 (Tyr705; p-STAT3) were from Cell Signaling Technology, Inc. (Beverly, MA, USA). Antibody specific for caspase-8 was from MBL. Antibodies against PARP-1 and SOCS7 were from Santa Cruz Biotechnology, Inc. (Santa Cruz, CA, USA). Anti- $\beta$-actin antibody was from Sigma (St. Louis, MO, USA).

Cells and cell culture. The human bladder cancer cell line T24 was obtained from Health Science Research Resources Bank (Osaka, Japan) and grown according to the manufacturer's protocol. The other human bladder cancer cell lines used, SNK57 and NKB1, ${ }^{32}$ were gifts from Cell Resource Center for Biomedical Research, Institute of Development, Aging and Cancer, Tohoku University and were maintained according to the recommended protocol. The number of viable cells was determined by performing the trypan blue dye exclusion test. Normal human urothelial cells (HUC) were purchased from ScienCell Research Laboratories (Carlsbad, CA, USA) and cultured according to manufacturer's protocol.
Transfections. Cells were seeded in 6-well or 12-well plate at a concentration of $0.5 \times 10^{5}$ cells per well the day before transfection. Transfection with miR-145, siRNA, PIC or plasmids was performed by using Lipofectamine RNAiMAX (Invitrogen) according to the manufacturer's protocol.

In cotransfection experiments with siR-socs7 and PIC, the cells were transfected with PIC at $48 \mathrm{~h}$ (T24) or $72 \mathrm{~h}$ (NKB1) after the siRNA transfection. The cells were then harvested at $24 \mathrm{~h}$ after the PIC transfection.

Western blotting. Total protein was extracted from whole cells by the procedure described previously. ${ }^{5}$ Protein contents were measured with a DC Protein Assay Kit (Bio-Rad, Hercules, CA, USA). Ten micrograms of lysate protein for western blotting was separated by SD S-PAGE using polyacrylamide gels and electroblotted onto a PVDF membrane (PerkinElmer Life Sciences, Boston, MA, USA). Details of the method used after blotting were described earlier. ${ }^{5}$ The loading control was prepared by re-incubating the same membrane with antihuman $\beta$-actin antibody.

Quantitative RT-PCR using real-time PCR. Total RNA was isolated from cells by the phenol/guanidium thiocyanate method with DNase I treatment. For determination of mRNA expression levels, total RNA was reverse transcribed with a PrimeScript RT Reagent Kit (TaKaRa, Otsu, Japan). Real-time PCR was then performed using SYBR Premix Ex Taq (TaKaRa). The relative expression level of mRNA was calculated by the $\triangle \triangle C t$ method. GAPDH was used as an internal control.

Assay for luciferase activity. We constructed the sensor vector by joining the region with a possible binding site from the $3^{\prime}$-UTR of human socs7 with a luciferase reporter pMIR-control vector (Ambion) to examine the target sequence recognized by miR-145. Moreover, to generate the sensor vectors with 2 mutations in the binding site for miR-145, we mutated seed regions from AACTGGAA to AATGAGAA (PrimeSTAR Mutagenesis Basal Kit; TaKaRa). The sensor vectors with mutations were submitted to Life Science Research Center, Gifu University for DNA sequencing. The cells were seeded in 12-well plates at a concentration of $0.5 \times 10^{5}$ per well the day before the transfection. The sensor vector (concentration; $0.5 \mu \mathrm{g} / \mathrm{well}$ ) and $40 \mathrm{nM}$ miR-145 or nonspecific control siRNA was used for the cotransfection of the cells. Forty-eight hours after the cotransfection, luciferase activities were measured by using a Dual-Glo Luciferase Assay System (Promega, Madison, WI, USA) according to the manufacturer's protocol. Firefly luciferase activity was normalized to Renilla luciferase activity.

Immunocytochemistry. T24 and NKB1 cells were plated on 13-mm glass coverslips (Matsunami Glass Ind., Osaka, Japan) in $1.0 \mathrm{ml}$ of culture medium plus $10 \%(\mathrm{w} / \mathrm{v})$ fetal bovine serum per well of a 6 -well plate. At $72 \mathrm{~h}$ after the transfection, cells were immunostained with anti-STAT3 antibody according to the immunofluorescence protocol of Cell Signaling Technology. The cells were viewed with a Biorevo fluorescence microscope (Keyence, Osaka, Japan).

Statistics. Each examination was performed in triplicate. The cell count analysis and the expression levels of mRNAs in the cells transfected with miR-145 and those transfected with control siRNA were compared by using Student's $t$-test. A $P$-value $<0.05$ was considered to be statistically significant.

\section{Conflict of Interest}

The authors declare no conflict of interest.

Acknowledgements. This work was supported by a grant-in-aid for scientific research from the Ministry of Education, Science, Sports, and Culture of Japan.

1. Ozen M, Creighton CJ, Ozdemir M, Ittmann M. Widespread deregulation of microRNA expression in human prostate cancer. Oncogene 2008; 27: 1788-1793.

2. Ichimi T, Enokida H, Okuno Y, Kunimoto R, Chiyomaru $T$, Kawamoto $\mathrm{K}$ et al. Identification of novel microRNA targets based on microRNA signatures in bladder cancer. Int J Cancer 2009; 125: 345-352

3. Akao Y, Nakagawa Y, Naoe T. MicroRNA-143 and -145 in colon cancer. DNA Cell Biol 2007; 26: 311-320.

4. Akao Y, Nakagawa $Y$, Kitade $Y$, Kinoshita T, Naoe T. Downregulation of microRNAs-143 and -145 in B-cell malignancies. Cancer Sci 2007; 98: 1914-1920. 
5. Noguchi S, Mori T, Hoshino Y, Maruo K, Yamada N, Kitade $Y$ et al. MicroRNA-143 functions as a tumor suppressor in human bladder cancer T24 cells. Cancer Lett 2011; 307: 211-220.

6. Noguchi S, Mori T, Hoshino Y, Yamada N, Nakagawa T, Sasaki N et al. Comparative study of anti-oncogenic microRNA-145 in canine and human malignant melanoma. J Vet Med Sci 2012; 74: 1-8.

7. Noguchi S, Yasui Y, Iwasaki J, Kumazaki M, Yamada N, Naito S et al. Replacement treatment with microRNA-143 and -145 induces synergistic inhibition of the growth of human bladder cancer cells by regulating PI3K/Akt and MAPK signaling pathways. Cancer Lett 2013; 328: 353-361.

8. Sachdeva M, Zhu S, Wu F, Wu H, Walia V, Kumar S et al. p53 represses c-Myc through induction of the tumor suppressor miR-145. Proc Natl Acad Sci USA 2009; 106: 3207-3212.

9. Chiyomaru T, Enokida H, Tatarano S, Kawahara K, Uchida Y, Nishiyama K et al. miR-145 and miR-133a function as tumour suppressors and directly regulate FSCN1 expression in bladder cancer. Br J Cancer 2010; 102: 883-891.

10. Gregersen LH, Jacobsen AB, Frankel LB, Wen J, Krogh A, Lund AH. MicroRNA-145 targets YES and STAT1 in colon cancer cells. PLoS One 2010; 5: e8836.

11. Xu N, Papagiannakopoulos T, Pan G, Thomson JA, Kosik KS. MicroRNA-145 regulates OCT4, SOX2, and KLF4 and represses pluripotency in human embryonic stem cells. Cell 2009; 137: 647-658.

12. Ostenfeld MS, Bramsen JB, Lamy P, Villadsen SB, Fristrup N, Sorensen KD et al. miR-145 induces caspase-dependent and -independent cell death in urothelial cancer cell lines with targeting of an expression signature present in Ta bladder tumors. Oncogene 2010; 29 1073-1084.

13. Sudbery I, Enright AJ, Fraser AG, Dunham I. Systematic analysis of off-target effects in an RNAi screen reveals microRNAs affecting sensitivity to TRAIL-induced apoptosis. BMC Genomics 2010; 11: 175

14. Zaman MS, Chen Y, Deng G, Shahryari V, Suh SO, Saini S et al. The functional significance of microRNA-145 in prostate cancer. Br J Cancer 2010; 103: 256-264.

15. Ludwig AT, Moore JM, Luo Y, Chen X, Saltsgaver NA, O'Donnell MA et al. Tumor necrosis factor-related apoptosis-inducing ligand: a novel mechanism for Bacillus Calmette-Guerininduced antitumor activity. Cancer Res 2004; 64: 3386-3390.

16. Chawla-Sarkar M, Leaman DW, Borden EC. Preferential induction of apoptosis by interferon (IFN)-beta compared with IFN-alpha2: correlation with TRAIL/Apo2L induction in melanoma cell lines. Clin Cancer Res 2001; 7: 1821-1831.

17. Doly J, Civas A, Navarro S, Uze G. Type I interferons: expression and signalization. Cell Mol Life Sci 1998; 54: 1109-1121.

18. Svoboda P. Off-targeting and other non-specific effects of RNAi experiments in mammalian cells. Curr Opin Mol Ther 2007; 9: 248-257.

19. Borden EC, Sen GC, Uze G, Silverman RH, Ransohoff RM, Foster GR et al. Interferons at age 50: past, current and future impact on biomedicine. Nat Rev Drug Discov 2007; 6 : 975-990.
20. Li G, Xiang Y, Sabapathy K, Silverman RH. An apoptotic signaling pathway in the interferon antiviral response mediated by RNase L and c-Jun NH2-terminal kinase. J Biol Chem 2004; 279: 1123-1131.

21. de Veer MJ, Holko M, Frevel M, Walker E, Der S, Paranjape JM et al. Functional classification of interferon-stimulated genes identified using microarrays. J Leukoc Biol 2001; 69: 912-920.

22. Dong B, Silverman RH. 2-5A-dependent RNase molecules dimerize during activation by 2-5A. J Biol Chem 1995; 270: 4133-4137.

23. Castelli JC, Hassel BA, Wood KA, Li XL, Amemiya K, Dalakas MC et al. A study of the interferon antiviral mechanism: apoptosis activation by the 2-5A system. J Exp Med 1997; 186: 967-972.

24. Krebs DL, Hilton DJ. SOCS proteins: negative regulators of cytokine signaling. Stem Cells 2001; 19: 378-387.

25. Martens N, Uzan G, Wery M, Hooghe R, Hooghe-Peters EL, Gertler A. Suppressor of cytokine signaling 7 inhibits prolactin, growth hormone, and leptin signaling by interacting with STAT5 or STAT3 and attenuating their nuclear translocation. J Biol Chem 2005; 280: 13817-13823.

26. Yang J, Chatterjee-Kishore M, Staugaitis SM, Nguyen H, Schlessinger K, Levy DE et al. Novel roles of unphosphorylated STAT3 in oncogenesis and transcriptional regulation. Cancer Res 2005; 65: 939-947.

27. Nepple KG, Aubert HA, Braasch MR, O'Donnell MA. Combination of BCG and interferon intravesical immunotherapy: an update. World J Urol 2009; 27: 343-346.

28. Yoneyama M, Kikuchi M, Natsukawa T, Shinobu N, Imaizumi T, Miyagishi M et al. The RNA helicase RIG-I has an essential function in double-stranded RNA-induced innate antiviral responses. Nat Immunol 2004; 5: 730-737.

29. Kato H, Takeuchi O, Sato S, Yoneyama M, Yamamoto M, Matsui K et al. Differential roles of MDA5 and RIG-I helicases in the recognition of RNA viruses. Nature 2006; 441: 101105.

30. Gitlin L, Barchet W, Gilfillan S, Cella M, Beutler B, Flavell RA et al. Essential role of mda-5 in type I IFN responses to polyriboinosinic:polyribocytidylic acid and encephalomyocarditis picornavirus. Proc Natl Acad Sci USA 2006; 103: 8459-8464.

31. Reich NC, Liu L. Tracking STAT nuclear traffic. Nat Rev Immunol 2006; 6: 602-612.

32. Naito S, Kotoh S, Koga H, Hasegawa S, Yamasaki T, Noma H et al. Establishment and characterization of two human bladder cancer cell lines. Hum Cell 1996; 9: 49-56.

Cell Death and Disease is an open-access journal published by Nature Publishing Group. This work is licensed under the Creative Commons Attribution-NonCommercial-No Derivative Works 3.0 Unported License. To view a copy of this license, visit http://creativecommons.org/licenses/by-nc-nd/3.0/ 